\title{
Asociación entre características sociodemográficas, estruc- tura familiar y embarazo en adolescentes
}

María C. Mejía G. ${ }^{1}$; Aníbal A. Teherán V. ${ }^{2}$; Mario de J. Serrano P. ${ }^{3}$; Lilian A. Andrews R. ${ }^{4}$; Paola A. Panesso P. ${ }^{5}$; Vanessa Cadavid ${ }^{6}$; María C. Barrera ${ }^{7}$

1 Médico y cirujano general. Centro de Investigación de la Fundación Universitaria Juan N. Corpas.

2 Médico especialista en epidemiología y estadística aplicada. Docente de investigación clínica, Centro de Investigación de la Fundación Universitaria Juan N. Corpas.

3 Médico especialista en epidemiología. Fundación Universitaria Juan N. Corpas.

4 Médico especialista en medicina familiar, Fundación Universitaria Juan N. Corpas.

5 Médico especialista en medicina familiar, Fundación Universitaria Juan N. Corpas.

6 Estudiante de medicina. Internado Rotatorio en Investigación. Fundación Universitaria Juan N. Corpas.

7 Estudiante de medicina. Internado Rotatorio en Investigación. Fundación Universitaria Juan N. Corpas.

\section{Correspondencia: Dra. María C. Mejía, maria-mejia@juanncorpas.edu.co \\ Como citar: Mejía MC, Teherán AA, Serra- no M, Andrews LA, Panesso PA, Cadavid V, Barrera MC. Asociación entre características sociodemográficas, estructura familiar y embarazo en adolescentes. Cuarzo 2015; 21 (2): $74-80$.}

Recibido: 15 de septiembre de 2015 Aceptado: 30 de noviembre de 2015. Publicado: 15 de diciembre de 2015.

Licencia creative commons c)

\section{Resumen}

Actualmente el embarazo en adolescentes es considerado un problema de salud pública que puede estar relacionado con características sociodemográficas y tipos de estructura familiar. En este artículo se presentan los resultados de una investigación que tuvo por objetivo evaluar la asociación entre características sociodemográficas, tipos de estructura familiar y embarazo en adolescentes, con el fin de proponer estrategias de intervención temprana; en esta se utilizó un estudio descriptivo retrospectivo, utilizando datos administrativos e información de registros médicos sobre adolescentes embarazadas, atendidas en una clínica de III nivel durante el periodo enero/diciembre-2014, y se analizó la frecuencia de características sociodemográficas y de tipos de estructura familiar para identificar relaciones entre estos dos aspectos. También se expone la necesidad de implementar planes multipropósito al momento de diseñar estrategias de prevención para este evento, con base en la interpretación de los resultados y las conclusiones de la investigación.

PALABRAS CLAVE: embarazo en adolescencia, clase social, datos demográficos, familia.

\section{Abstract}

\section{Association between sociodemographic characteristics, fa- mily structure and pregnancy in adolescents}

Currently teen pregnancy is considered a public health problem that may be related to sociodemographic characteristics and types of family structure. This article presents the results of an investigation that aimed to assess the association between sociodemographic characteristics, types of family structure and adolescent pregnancy, in order to propose early intervention strategies are presented; in this retrospective descriptive study it was used, using administrative data and information from medical records of pregnant teenagers attending a clinic III level during the period January / December 2014, 
and the frequency of sociodemographic characteristics and types of structure was analyzed family to identify relationships between these two aspects. It also exposes the need to implement multipurpose plans when designing prevention strategies for this event, based on the interpretation of the results and conclusions of the investigation.

KEYWORDS: Pregnancy in Adolescence, Family, demographic data, social class.

\section{Introduction}

La Organización Mundial de la Salud (OMS) define embarazo en adolescentes o precoz como el gestado en mujeres entre 10-19 años de edad (1). Es un evento de salud pública asociado a elevadas tasas de morbilidad y mortalidad en la madre y el neonato (2); además, genera impactos socioculturales y psicológicos negativos, con elevados costos a nivel personal, educacional y familiar (3).

El embarazo en adolescentes representa $11 \%$ de nacimientos a nivel mundial y $19 \%$ en países en vía de desarrollo (1, 4). En Colombia, en el año 2010, la frecuencia de adolescentes que alguna vez estuvieron embarazadas fue $22,1 \%, 66 \%$ de estos embarazos no fueron planeados y con relación al 2005, la ocurrencia de embarazos en adolescentes disminuyó sólo 1\%; anualmente, esto cuesta 810.562 millones de pesos para el Sistema General de Seguridad Social en Salud y, para el 2014, se estimó una tasa de fecundidad de 52/1000 mujeres (5-9). La relevancia de estos datos radica en que se estima que la población de mujeres adolescentes en Bogotá es cercana a 960.537 personas, y aproximadamente 2 de cada 10 ha estado embarazada; esto se suma a que cerca del $10 \%$ de las adolescentes entre 11-14 años de Bogotá y más de un tercio de aquellas entre 15-16 años durante el 2013 no estudiaban, factor fuertemente asociado con la ocurrencia de embarazos en la adolescencia (10-12). Existen diferentes tipos de factores de riesgo para embarazo en adolescentes, por ejemplo, sociodemográficos: nivel socioeconómico y educativo de los padres y adolescentes (13); clínicos: drogadicción y antecedentes patológicos de la paciente, y relacionados con la estructura familiar: principalmente condiciones que alteran el núcleo primario, entre ellas, disolución de la familia a causa de divorcios y las muertes o ausencia de uno de los padres $(14,15)$.

Estudios locales han demostrado asociación entre factores de riesgo sociodemográficos y han descrito que los factores relacionados con la estructura familiar son fun- damentales, dado que, a través de sus miembros y las relaciones entre ellos, se imprimen componentes esenciales en el desarrollo de los individuos y se ha reconocido que la familia y su estructura influyen directamente en el comportamiento sexual de las adolescentes. Por ejemplo, la crianza en familias monoparentales se ha relacionado con embarazos en esta etapa de la vida por el inicio de la actividad sexual temprana, al parecer por la necesidad de buscar compensaciones emocionales en la actividad sexual, que reemplacen la falta de afecto de uno de los miembros del núcleo (16-18).

Con base en lo anterior se realizó una investigación en la que se evaluó la asociación entre factores de riesgo sociodemográficos, características de la estructura familiar y embarazo en adolescentes, con el fin de proponer estrategias de intervención temprana en adolescentes con alto riesgo de embarazo, y cuyos resultados se exponen en este artículo.

\section{Metodología}

Para la selección de los pacientes, se realizó un estudio descriptivo retrospectivo, con una muestra por conveniencia de adolescentes embarazadas, atendidas en el servicio de ginecología de una clínica de III nivel de atención, durante el periodo enero/2014-diciembre/2014.

El departamento de Bioestadística de la clínica suministró una base de datos con 1055 pacientes, se incluyeron mujeres entre 10 y 19 años con historias clínicas completas y se excluyeron 47 por no cumplir con los criterios de elegibilidad establecidos.

Los análisis y reportes de la investigación fueron realizados basados en la guía metodológica Strenghtening the Reporting of Observational Studies in Epidemiology (STROBE) (19).

La base de datos original contenía información sociodemográfica, fecha y servicio de atención, que fue complementada con datos de los componentes de la estructura 
familiar y de otras características clínicas como el uso de drogas, obtenidos a partir de la valoración psicológica contenida en los registros médicos de atención. En los casos en los que no dispuso de la valoración psicológica, se utilizó la información consignada por médicos y enfermeras en los controles prenatales o del ingreso a urgencias/ hospitalización el día del parto; el número de identificación de las adolescentes se cambió por un consecutivo y la base de datos fue analizada por personal no tratante, con el fin de garantizar el anonimato de las pacientes (https:// drive.google.com/drive/u/0/folders/0B0BzWSj_ ng8CQjBoLTJsRTFyRUU).

Las variables sociodemográficas incluidas fueron: edad, estado civil, estrato socioeconómico (ES) clasificado según lo establecido por el Departamento Administrativo Nacional de Estadística y reagrupado en bajo [1-2], medio [3-4] y alto [5-6]; el nivel educativo fue reagrupado en $\leq$ primaria, bachiller/técnico $\mathrm{y} \geq$ profesional (15).

Las familias se clasificaron en nuclear (vive con padres y hermanos), monoparental (vive con padre o madre), nuclear extensa (familia nuclear y otros miembros de la familia como los abuelos) y reconstituida (uno de los padres con un padrastro o madrastra); además se indagó sobre la condición de abandono social (sin cuidado parental) $(20,21)$.

Se evaluó la presencia de enfermedades preexistentes como una forma indirecta de indagar el uso de medicamentos que disminuyeran la eficacia de los anticonceptivos; además, se preguntó sobre el uso de drogas, entre ellas, el alcohol, tabaco u otras sustancias psicoactivas diferentes a las anteriores, de manera general o individualizada para cada una de las mencionadas.

La variable resultado primaria fue: frecuencia de tipos de estructura familiar y las características sociodemográficas en adolescentes embarazadas; y la secundaria: la relación entre factores de riesgo de tipo sociodemográficos y tipos de estructura familiar en adolescentes embarazadas.

Los datos se analizaron con los softwares Minitab ${ }^{\circledR}$ (Versión 16) y Epilnfo®, (Versión 3.5.4) y se expresaron en medias (IC95\%), desviaciones estándar y proporciones.

Las comparaciones entre datos continuos, para dos grupos independientes, se realizaron con una prue ba $\mathrm{T}$ de Student. Los datos categóricos se compararon con la prueba de Chi-cuadrado. Se estableció una $\mathrm{p}<0,05$ (dos colas) como significativa. Con una tabla de $2 \times 2$ se estimó el OR (IC95\%) para determinar la relación entre cada uno de los tipos de estructura familiar y las variables sociodemográficas.

\section{Resultados}

Durante el periodo estudiado se incluyeron 1008 adolescentes, caracterizadas por edad de $17 \pm 1$ años, 94\% solteras, $78,7 \%$ pertenecían a ES bajo; $48,5 \%$ solo finalizó primaria. El nivel educativo de los padres fue encontrado en $47 \%$ de los registros, en estos, la mayoría cursó hasta primaria o bachillerato (87,8\%; IC95\%, 85,1-90,5\%); menos del 5\% de las adolescentes tenían antecedentes de una condición patológica (tabla 1).

El antecedente de uso de sustancias psicoactivas (general o individual), la convivencia en familias monoparentales y el nivel de educación de educación del padre ( $\leq$ primaria) se relacionaron con embarazos a edades más tempranas $(\mathrm{p}<0,05)$ (figura 1$)$.

Comparación de edad media (IC95\%) al momento de la atención. Las medias representadas con cuadros rellenos identifican los factores relacionados con edades más tempranas al momento del embarazo, las representados con círculos rellenos aquellos relacionados con embarazos más tardíos. Otras sust: otras sustancias psicoactivas diferentes al alcohol-tabaco; Drogas: antecedente en general de uso de drogas (alcohol, tabaco, otras sustancias psicoactivas); Educ_padre_0: padre sin educación o $\leq$ primaria; Educ_padre_1: padre con educación $\geq$ bachiller; Nuclear: familia de tipo nuclear; Monoparen_: familia de tipo monoparental. Fuente: elaboración propia.

\section{Distribución de características relacionadas con la estructura familiar}

La mayoría de adolescentes pertenecían a una familia de tipo nuclear $(54,6 \%$; IC95\%, 51,4- 57,7\%), seguido de familias no nucleares (45,4\%IC95\% 42,3-48,6\%); entre estas, se identificaron diferencias con respecto a la media de edad y al ES medio-bajo. Las adolescentes que pertenecían a familias de tipo nuclear tenían edad media mayor que las pertenecientes a familias no nucleares $(0,3$; IC95\%, 0,14-0,46); se encontró mayor frecuencia de adolescentes pertenecientes al ES medio, que convivían en familias nucleares (7,5\%; IC95\%, 2,5-12,5\%), a diferencia de aquellas que pertenecían al ES bajo quienes, con mayor frecuencia, convivían en familias no nucleares (7,8\%; IC95\%, 2,8-12,8\%) (tabla 2). 
Los otros tipos de familia identificados, diferentes a la nuclear fueron: nuclear extensa 17,6\% (IC95\%, 15,219,9\%), monoparental 13,8\% (IC95\%, 11,6-15,9\%), reconstituida $84 \%$ (IC95\%, 6,7-10,2\%) y en condición de abandono social 2,4\% (IC95\%, 1,4-3,4\%).

\section{Características sociodemográficas o clínicas y tipos de estructura familiar en adolescentes embarazadas}

En un análisis exploratorio preliminar se evaluaron posibles relaciones entre los tipos de familia y las características sociodemográficas o clínicas (tabla 3).

Posteriormente, entre las parejas de características sociodemográficas y los tipos de familia que, preliminarmente presentaron asociaciones estadísticas $(p<0,05)$, se determinó la magnitud de la relación (tabla 4).

Se encontró una fuerte relación entre: i) la pertenencia al ES medio y la convivencia dentro de familias nucleares, ii) el consumo de otras sustancias psicoactivas (diferentes al tabaco-alcohol) y la convivencia dentro de familias monoparentales, iii) el estado civil de soltería con la convivencia en familias nucleares extensas y iv) los estados civiles unión libre o casada con el antecedente de abandono social.

\section{Conclusiones y discusión}

La investigación permitió evidenciar fuertes nexos entre factores de riesgo sociodemográficos y características de la estructura familiar, para el embarazo en adolescentes. El contexto social donde se ejecutó esta investigación está conformado por familias pertenecientes a ES bajos o medios y $70 \%$ de estas no tienen cobertura para los gastos mínimos del hogar (22). En la mayoría de hogares, los padres trabajan fuera de casa, factor que limita la supervisión de comportamientos de alto riesgo sexual, aún en los adolescentes que conviven bajo el amparo de una familia nuclear; este riesgo se puede reducir en familias pertenecientes a ES altos, debido al cuidado exclusivo que aporta uno de los padres al hogar, característica que libera de responsabilidades económicas al cónyuge.

El perfil demográfico de adolescentes embarazadas es consistente con lo descrito en investigaciones previas, la mayoría de hogares pertenecía al ES bajo, y tanto padres, como adolescentes, tenían bajo nivel educativo. Este patrón es repeti tivo y no difiere en países con bajos, medianos o altos ingresos económicos $(22,23)$.

En este subgrupo específico de embarazadas, que transitan en la etapa adolescente, se encontró relación, aunque débil, entre la convivencia dentro de familias monoparentales y la pertenencia a un ES bajo; asimismo, la convivencia dentro de este tipo de estructura familiar evidenció fuertes nexos con el consumo de otras sustancias psicoactivas diferentes al tabaco-alcohol y con un nivel educativo bajo de las mencionadas. Estos hallazgos son consistentes con la explicación planteada, sobre la falta de supervisión de alguno de los padres en hogares con bajos ingresos económicos, que podría exponer a la adolescente a conductas de riesgo de tipo sexual y uso/abuso de sustancias psicoactivas; el bajo nivel educativo puede explicarse por la ocurrencia de embarazos a edades más tempranas en los subgrupos de adolescentes que consumían otras sustancias psicoactivas y que convivían en familias de tipo monoparental $(17,18,24)$. Otra explicación sobreviene por limitaciones presentes en las adolescentes, dentro del marco de la crianza en familias monoparentales, con bajos recursos económicos, donde las embarazadas tienen bajos niveles educativos, con escasos conocimientos sobre métodos de planificación y no disponen de soporte económico para acceder a los anticonceptivos más eficaces $(25,26)$.

Un hallazgo novedoso fue la identificación de vínculos entre el ES medio y la convivencia en familias nucleares; frecuentemente y de manera independiente, se ha identificado que, pertenecer al ES bajo y ocasionalmente al ES medio son factores de riesgo para embarazos en adolescentes $(20,27,28)$. Un estudio realizado en Portugal evidenció que, en las adolescentes embarazadas, existía una relación entre la pertenencia al ES bajo y la convivencia en familias nucleares; cabe aclarar que, la escala implementada para clasificar los ES en este país difiere de la nuestra y que en la metodología los investigadores reclasificaron estos niveles de una escala de 5 a 2 categorías, estrategia que limita las comparaciones con nuestros resultados (22).

En población ecuatoriana se evidenció que los factores relacionados con la presentación de embarazos a edades muy tempranas se agrupan en aquellos de tipo compor- 
tamental, como inicio temprano de vida sexual y no uso de anticonceptivos durante la primera relación y otros de tipo estructural, como ES medio o bajo, antecedente de abuso sexual durante la infancia/adolescencia y determinadas características de la estructura familiar (30). Nuestros resultados evidenciaron coincidencias con los factores mencionados, pero a diferencia del estudio realizado en Ecuador, que tenía como variable resultado el riesgo de un embarazo en la adolescencia, en nuestro estudio todas las participantes estaban embarazadas y los complejos multifactoriales fue nuestra variable resultado.

Esta investigación presenta algunas limitaciones teóricas entre ellas: i) la información fue obtenida al final del embarazo, y entre el inicio y el final de este período, las condiciones socioeconómicas y el tipo de estructura familiar pudo cambiar, ii) no se obtuvo información sobre los métodos de planificación o el contenido de educación sexual recibida por las adolescentes, factores influyentes en la ocurrencia del embarazo en la adolescencia, iii) se desconocían datos sobre la edad de parejas (novios-cónyuges) o de embarazo de las madres y ocupación de los padres, variables que también han evidenciado relación con este evento, y iv) ausencia de un grupo de comparación que debe contemplarse en futuras investigaciones. Cabe mencionar que, las anteriores limitaciones son frecuentemente encontradas en diseños metodológicos con inicio cronológico en variedad retrospectiva en los que, a excepción de aquellos que utilizan instrumentos tipo cuestionario y logran identificar a los participantes, solo se cuenta con la información contenida en los registros de atención médica.

Los resultados obtenidos permiten concluir que en adolescentes embarazadas existen vínculos fuertes entre características sociodemográficas, previamente identificadas como factores de riesgo en la literatura y algunos tipos de estructura familiar. En el contexto colombiano que, en la última década ha presentado un aumento del $30 \%$ en la tasa de fecundidad, los resultados de esta investigación que colocan de manifiesto la interacción entre componentes sociodemográficos y del tipo de estructura familiar, nos permiten reflexionar acerca de las estrategias de promoción y prevención que hemos adoptado para disminuir la tasa de embarazo en adolescentes (9). Desde el año 2012, se tomaron determinaciones en materia de salud pública con el fin de disminuir este fenómeno crecien- te en América Latina pero agudizado en Colombia; entre las estrategias planteadas era relevante la necesidad de un abordaje multifactorial, que permitiera de manera integral intra y transectorial convocar a todos los organismos que pudiesen intervenir en el desarrollo de los lineamientos establecidos.

Se estableció que, el concepto de "resiliencia" deberá formar parte de los cimientos y la planeación estratégica para la prevención del embarazo en adolescentes, entendiendo este como la interacción entre los determinantes próximos, intermedios y distales que aumentan la probabilidad de ocurrencia del evento, a pesar de que en ese tiempo la evidencia científica de Colombia identificaba a estos factores de riesgo de manera independiente para el embarazo en adolescentes, fue muy acertado su abordaje de manera integral (9).

Los resultados de la investigación demuestran que dicho pensamiento no estaba alejado de la realidad y que la manera de abordar esta problemática era concordante con lo sugerido por entes internacionales $(31,32)$; por lo anterior, se recomienda hacer énfasis en estas estrategias multipropósito para mejorar la situación del problema y de esta manera se evidenciaran los cambios, fruto de las políticas adoptadas.

\section{Referencias}

1. Organización Mundial de la Salud [internet]. Ginebra: OMS; 2016 [actualizada enero de 2016; acceso 8 de junio de 2016]. Disponible en: http://www.who.int/mediacentre/ factsheets/fs364/es/

2. Arcelus M. La adolescente embarazada y su familia. El embarazo en la adolescente. México, DF. 1998; 22 15-20.

3. De Rodríguez Isabel Cluet, Rossell-Pineda María del Rosario, Álvarez de Acosta Thais, Rojas Quintero Ligia. Factores de riesgo asociados a la prematuridad en recién nacidos de madres adolescentes. Rev Obstet Ginecol Venez [Internet]. 2013 [acceso 08 de junio de 2016]; 73(3): [157-170]. Disponible en: http:// www.scielo.org.ve/scielo.php?script=sci_ arttext\&pid=S0048$77322013000300003 \& \operatorname{lng}=\mathrm{es}$

4. Marfan J, Córdoba C. La educación sexual en América Latina: revisión de algunos programas educativos en la región. Documento presentado al Consejo Episcopal Latinoamericano (CELAM). Reduc.cl [revista en internet] 2014 [acceso 08 de junio de 2016]. Disponible en: http://www. reduc.cl/reduc/marfan.pdf

5. Grupo Banco Mundial [sede Web]. BM; 2016 [15 de Junio de 2016; acceso 15 de junio de 2016].Tasa de fertilidad en Adolescentes. Disponible en: http://datos.bancomundial.org/indicador/ SP.ADO.TFRT?order=wbapi_data_value_2014+wbapi_data value+wbapi_data_value-last\&sort $=$ asc 
6. Centro Latinoamericano de Salud y Mujer [internet]. México: CELSAM; 2001-[actualizada enero de 2016; acceso 8 de junio de 2016]. Disponible en: http://www.yourlife. com/es/para-medicospadres-etc/acerca-del-dma/centrolatinoamericano- de-salud-ymujer-celsam/\#

7. Ojeda G, Ordóñez M, Ochoa L. Encuesta Nacional de Demografía y Salud 2010. $1^{\text {a }}$ ed. Bogotá: Profamilia; 2011.

8. Ojeda G, Ordóñez M, Ochoa L. Encuesta Nacional de Demografía y Salud 2010. $1^{\text {a }}$ ed. Bogotá: Profamilia; 2005.

9. Consejo Nacional de Política Económica y Social República de Colombia Departamento Nacional de Planeación. Lineamientos para el desarrollo de una estrategia para la prevención del embarazo en la adolescencia y la promoción de proyectos de vida para los niños, niñas, adolescentes y jóvenes en edades entre 6 y 19 años. CONPES 147. Disponible en: http://periodico.sena.edu.co/ descargables/ Conpes\%20social\%20147\%20(2).pdf

10. Secretaria Distrital de Planeación de Bogotá. Adolescentes en Bogotá. Participación y Derechos. Bogotá 2014; 4,21. Disponible en http://www.sdp.gov.co/portal/page/ portal/PortalSDP/ InformacionTomaDecisiones/Estadisticas/ Bogot\%E1\%20Ciudad\%20de\%20Estad\%EDsticas/2014/ Estudio_Adoloescentesjulio2014-62.pdf

11. Secretaria de Educación. Caracterización Sector Educativo. Bogotá; 2013. [acceso 15 de junio de 2016] Disponible en: http:// www.educacionbogota.edu.co/archivos/SECTOR_ EDUCATIVO/ESTADISTICAS_EDUCATIVAS/2013/BoletinEstadisticoAnual2013. pdf

12. Departamento Administrativo Nacional de Estadísticas. DANE. Proyección de población Jovenes. [junio 16 de 2016, último acceso junio 16 de 2016]. Disponible en: http://obs.colombiajoven.gov.co/ Observatorio/Observatorio. aspx?rpt=Buyel7PqAptVw91wUXO_ $\mathrm{Fw}==$

13. Patton GC, Coffey C, Sawyer SM, Vinner RM, Haller DM, Bose $\mathrm{K}$ et al. Global patterns of mortality in young people: a systematic analysis of population health data. Lancet [revista en Internet] 2009 [acceso 8 de junio de 2016]; 374(9693): [881-92]. Disponible en: http://www. thelancet.com/pdfs/journals/lancet/PIIS01406736- (09)60741-8.pdf

14. Sánchez L, Gutiérrez ME, Herrera N, Ballesteros M, Izzedin R, Gómez A. Social representations of having a boy/ girlfriend in adolescents still in school from low, middle and high social strata in Bogotá. Rev Salud Publica (Bogota). 2011 Feb;13(1):79-88.

15. Smetana J. Family structure parental monitoring and adolescent problem behavior middle-class African American Families. J Res Adolesc [revista en Internet] 2002 [acceso 8 de junio de 2016]; 12(2): [275-304]. Disponible en: https:// www.researchgate.net/ publication/229475126_Family_Processes_and_Problem_Behaviors_in_Middle-Class_African_American_Adōescents

16. Hernández A. Estrés en la familia colombiana: tensiones típicas y estrategias de afrontamiento [tesis doctoral]. Bogotá: Universidad Santo Tomás; 1991.
17. Quigley A. Father's Absence Increases Daughter's Risk of Teen Pregnancy. Health Behavior News Service [revista en Internet] 2003 [acceso 08 de junio de 2016]: [19-98]. Disponible en: http:// www.cfah.org/hbns/news/daughter05- 14-03.cfm

18. Steinberg L, Lamborn L, Dornbusch SM, Darling N. Impact of Parenting Practices on Adolescent Achievement: Authoritative Parenting, School Involvement, and Encouragement to Succeed. Child Dev [revista en Internet]. 1992 [acceso 08 de junio de 2016]; 63(5): [1266-81]. Disponible en: http://onlinelibrary.wiley. com/doi/10.1111/j.1467-8624.1992.tb01694.x/ epdf?r3_ referer=wol\&tracking_action=preview_click\&show_ checkout $=1 \&$ purchase_referrer=www.ncbi.nlm.nih. gov\&purchase_site_license=LICENSE_DENIED

19. Von Elm E, Altman DG, Egger M, Pocock SJ, Gøtzsche PC, Vandenbroucke JP, et al. The Strengthening the Reporting of Observational Studies in Epidemiology (STROBE) Statement: Guidelines for Reporting Observational Studies. Ann Intern Med [revista en Internet]. 2007 [acceso 08 de junio de 2016]; 335(7624): [806-8]. Disponible en: http://www.bmj.com/ content/335/7624/806.long

20. Departamento Administrativo Nacional de Estadística DANE [sede Web]. Colombia: DANE; 2016 [09 de junio de 2016; acceso 12 de junio de 2016]. Estratificación socioeconómica [aproximadamente 3 pantallas]. Disponible en: http:// http:// www.dane.gov.co/index.php/estratificacion- socioeconomica/ generalidades\#sgpbl_4

21. Santos M, Rosário F. A score for assesing the risk of first-time adolescent pregnancy. Family Practice 2011;28:482-488.

22. Durán E, Valoyes E. Perfil de los niños, niñas y adolescentes sin cuidado parental en Colombia. Rev.latinoam. cienc.soc.niñez juv [Internet]. 2009 July [cited 2016 June 13];7(2)761-783. Available from: http://www.scielo. org.co/scielo.php?script=sci_ arttext\&pid=S1692-715- X2009000200008\&lng=en

23. Valdivia M, Molina M. Factores Psicológicos Asociados a la Maternidad Adolescente en Menores de 15 años. Rev. psicol [revista en internet]. 2003; 12(2): [85-109]. Disponible en: http:// www.revistapsicologia.uchile.cl/index. php/RDP/article/viewFile/17457/18228

24. Pradhan R, Wynter K, Fisher J. Factors associated with pregnancy among adolescents in low-income and lower middleincome countries: a systematic review. J Epidemiol Community Health [revista en Internet] 2015 [acceso 08 de junio de 2016]; 69(9): [918-24]. Disponible en: http://jech. bmj.com/content/ early/2015/06/01/jech-2014-205128.

25. Slater J. Britain: Sex education under fire. Unesco Cour [revista en Internet] 2000 [acceso 08 de junio de 2016]; 53(7): [17-18]. Disponible en: http://unesdoc.unesco.org/ images/0012/001201/120152e.pdf

26. Komura L. Adolescent maternity in a low income community: experiences revealed by oral history. Rev Latino-am Enfermagem 2008;16(2):280-6. 
27. Ministerio de la Protección Social. Consejo Nacional de Seguridad Social en Salud. 2007. [acceso junio 15 de 2016]. Disponible en: http://www.sociedadescientificas.com/userfiles/ file/ACUERDOS/380\%2007.pdf

28. CEPAL. Cambio de las familias en el marco de las transformaciones globales: necesidad de políticas públicas eficaces. [monografía en Internet]. Santiago de Chile: Naciones Unidas; 2004 [acceso 09 de junio de 2016]. Disponible en: http://repositorio. cepal. org/bitstream/handle/11362/6773/1/S0412955_es.pdf

29. Imamura M, Tucker J, Hannaford P, et al. Factors associated with teenage pregnancy in the European Union countries: a systematic review. European Journal of Public Health 2007; 17(6):630-636.
30. Goicolea I, Marianne W, Öhman A, San Sebastian M. Risk factors for pregnancy among adolescent girls in Ecuador's Amazon basin: a case-control study. Rev Panam Salud Publica [Internet]. 2009 Sep [cited 2016 June 13] ; 26 ( 3 ): 221-228. Available from: http:// www. scielosp.org/scielo.php?script=sci_arttext\&pid=S102049892009000900006\&lng=en. http://dx.doi.org/10.1590/ S102049892009000900006.

31. Chandra-Mouli V, Camacho A. WHO Guidelines on Preventing aarly Pregnancy and Poor Reproductive. Journal of Adolescent Health. 2013: 517-522.

32. Kelsey M, Layzer J. Implementing Three Evidence- Based Program Models: Early lessons from the Teen Pregnancy Prevention Replication Study. Jurnal of Adolescent Health. 2014. 54:S45-S52. 